\title{
Designing with Midwives: Improving Prenatal Care in Low Resource Regions
}

\author{
Abdullah Al Mahmud \\ ID-StudioLab, Industrial Design Engineering \\ Delft University of Technology \\ 2628CE Delft, the Netherlands \\ a.almahmud@tudelft.nl
}

\author{
David V. Keyson \\ ID-StudioLab, Industrial Design Engineering \\ Delft University of Technology \\ 2628CE Delft, the Netherlands \\ d.keyson@tudelft.nl
}

\begin{abstract}
This paper describes the early design of a diagnostic system to support prenatal care of rural pregnant women. Our design is based on interviews and feedback from midwives who have worked in developed and developing countries. Based on the interviews we identified key issues in prenatal care and properties of a basic diagnostic system applicable in a low resource setting. The design requirements and challenges were verified with midwives and a concept was developed. Furthermore, we report technical requirements and limitations to implement the proposed diagnostic system in a rural setting.
\end{abstract}

Keywords-prenatal care, developing countries, diagnostic support system, midwife, maternal health

\section{INTRODUCTION}

Each year worldwide 529000 maternal death occur [2]. The target set for MDG-5 is a 75\% reduction of maternal mortality ratio between 1990 and 2015. The reason is partly due to poor diagnostic capability and on the other hand pregnancies are often not reported for such cases. The ongoing high maternal mortality in low-resource countries shows that there is a need to identify and implement strategies that are most effective to reduce maternal mortality [3]. There are three main approaches of safe motherhood strategies and the phases are classified as pregnancy, antenatal, delivery and postpartum period [4]. Prenatal care consists of a number of interventions administered to women during pregnancy, including screening tests, immunizations, and treatment for identified complications. It has been established that the detection of early signs or risk factors for, morbidity and mortality can be detected and effective interventions are possible [7]. Appropriate technology such as simpler clinical diagnostic tool may help at primary healthcare level to improve antenatal care.

In this pilot project we investigate the possibility to design a tool for rural healthcare workers to support antenatal care. The diagnostic support system has the following objectives: a) improving point of care diagnostic support, b) screen for patients who need to visit a physician and c) provide advice/tips for those who do not need to visit a physician or an expert. A user centered design approach has been followed in this project. The design process is divided into three phases: inspiration, concept creation and evaluation. Key design aspects for the diagnostic support system were identified from the interviews. The design aspects were verified and refined after consultation with experienced midwives in the Netherlands. Furthermore, feasibility of the generated concepts were evaluated against criteria such as technology, cost, manpower that are available in low-resource settings. In the following sections at first we describe related work and field studies results. Later on we present the key design implications and implementation details of the proposed system.

\section{RELATED WORK}

There are a couple of ongoing projects that aim to improve antenatal care in developing countries for example MAMA project in Bangladesh [12]. In this pilot project mobile health messages are designed to provide information that will inform women regarding a) care for themselves during pregnancy, b) make new mothers aware of how best to care for their babies, c) give reminder for immunization, d) advise about nutrition of mother and child, etc. Mobile message have been used effectively to promote maternal health in other countries like India [11]. Besides these there are some applications which are particularly designed for diagnostic support for pregnant women. One notable example is the 'click diagnostic' application. The application has been designed to support maternal, neonatal and child health. The basic idea of the application is that community health workers collect data about pregnant women and send to a specialist. The specialist view the patient information and provide feedback to the cell phone of the community health workers [1]. This is a promising application however, many places in developing countries do not have any internet and there are shortage of rural healthcare workers. Another application called 'Early Detection and Prevention System (EDPS)' developed in India by the George Foundation. The systems has been used in India for eight years and allows people without extensive training to help diagnose illness [5]. The system has 94\% accuracy record which is equivalent to diagnose of an experienced doctor. However, the system has not been designed to support prenatal care rather to support epidemiological studies. A web-based clinical diagnostic system to improve antenatal care has been used in Brazil and the system is called SUAP (prenatal care unified system). SUAP uses agent technology to manage healthcare records, to act as a clinical decision support system and to handle the logistics of high risk pregnancy cases [7]. The project is still ongoing and cannot be directly implemented in a low-resource environment since it was designed to facilitate information processing and to support medical professionals to 
accomplish tasks regarding clinical protocols. In the following section we explain the field studies and results.

\section{FILED STUDIES \& RESULTS}

In total 12 midwives participated in a semi-structured interview. The midwives were based in the Netherlands and they had experience in working in developing countries like Pakistan, India and African countries like Tanzania, Zambia, etc. The preliminary questions for the interviews were a) key issues related to participation in prenatal care in low resource environments, b) IT knowledge and support for rural health workers, c) properties of a basic diagnostic system applicable in a low resource setting, and d) factors associated with the success of a basic diagnostic system. Each interview session was one hour long. Interview data were audio recorded and later transcribed in English. The data were thematically analyzed by the first author and one external researcher and the method described in Grounded Theory [10] was followed. The key themes emerged from the interviews are explained below.

\section{A. Issues related to low participation in the prenatal care}

Several issues were identified during the interviews regarding low participation in prenatal care. The key issues are 1) lack of knowledge, 2) cost of care, 3) social barriers to care, and 4) displeasure with medical facilities. The crucial reason is lack of knowledge about prenatal care and general education. Sometimes pregnant women cannot afford to go for costly medical checkup during pregnancy in a hospital. One midwife (MW3) mentioned, "I often see that antenatal care is missed out, people often do not come for antenatal care. The reason is often money that they have to pay for care. Sometimes women make their own choice because they just do not want to go to hospital". Another midwife mentioned (MW7), "sometimes women do not come because they hear that hospital is not a nice place, so even they can pay they do not come". It has been observed by the midwives that women who already have children they come to the hospital willingly. MW1 mentioned, "sometimes if they have children they come to the hospital, you could say look you can come for the vaccination of your child and at the same time you can get antenatal care which is a handy way of doing it. But women who are pregnant for the first time are the most difficult one". In some places women are not allowed to go to hospital without permission from the village chief/elders. Elders in the village need to know that it is important to go to hospital and then allow them to go.

\section{B. IT knowledge and support for rural health workers}

Two key issues were identified in this area such as 1) tech literacy and 2) illiteracy. Midwives mentioned that in most cases rural health workers are not computer literate and there are many languages in rural areas. M8 mentioned, "healthcare workers say that they are computer literate, because they feel a lot of pride, but they are not. So you have to be very careful that you do not hurt their pride. However many people are very mobile literate and they can use a mobile phone very well. But you need to see who are the real people and who will take care of the pregnant women and you need to find a system for them. If they cannot read or write, the system should provide auditory support so that the care provider can hear in their own language”. Therefore, it is very important to consider the capabilities of people who provide care in low resource settings. There are villages where there is no electricity supply regularly and no pure water but they have mobile phones and they have 3G in the air. Therefore, it is a positive fact that these aspects can be utilized for better prenatal care support.

\section{Properties of a basic diagnostic system applicable in a low resource setting}

Key issues identified in this area were 1) design challenges, and 2) key features of a diagnostic support system. All the interview participants advised to develop a system which is very basic, easy to operate and understand. One midwife (MW10) mentioned, “do not put your targets too high. If you can manage an antenatal software support system by which women who are pregnant are visited 4 times, with a few basic things like feeling the baby, measuring the blood pressure, etc. is sufficient and I will congratulate you. Sometimes just talking to the women with basic tips is supportive and if you look at the women and she trust you that is very helpful than anything". Since the system will be used in a low resource setting identifying the basic health problems during pregnancy along with family history would be the first step. For example thalassemia/anemia which are more common in low resource areas like in India and Bangladesh and the system should be able to identify and provide advise based on these diseases. MW2 mentioned. "find out what are the causes of fetal and maternal death in the region. In your system you can set rules like, if you have twins: go to hospital, if the baby's head is upside: go to hospital, if the baby is too big: go to hospital, these are the very basic things that the software system should support". Secondly, the system should have capability to provide advice on food and nutrition based on personal situation of the pregnant women. In developing countries especially in remote villages people have less knowledge about nutritious food. Therefore, the diagnostic system should have capability to suggest healthy foods based on local situation and affordability of the mother. Furthermore, the system should have capability to indicate what kind of vitamin supplements the pregnant women should take over the 9 months. In addition risky behaviors and recommended exercises should be advised by the diagnostic system. Since the number of specialized doctors are not many in low resource environments the system should provide suggestions when to visit a specialist. Furthermore, some basic point of care testing devices such as blood pressure measurement, blood glucose measurement as well as devices like Doppler machine and cheap ultrasound machine would be really helpful. In that case the diagnostic system should have capability to process the data gathered by the point of care testing devices and provide suggestions to pregnant woman individually. Other positive suggestion was that the system should encourage pregnant women to go to the local hospital for giving birth since there will be less skilled birth attendants in the village. 


\section{Factors associated with the acceptance of a rural diagnostic system}

We identified two factors such as 1) social hierarchy and 2) cultural barriers and preferences that may hinder the acceptance of a rural diagnostic system. One of the key issues identified was to include all the people involved in providing care for a pregnant women such as from the traditional birth attendant to community health workers. MW1 mentioned, "you need to see who has the power and have to keep them in the loop. For example, you need to include traditional birth attendants in the system. Include all the different people because they have a different role to play. Find a way so that all persons providing care also get respect since sometimes they find themselves not treated properly by other colleagues who are upper position in the hierarchy." It was revealed that the acceptance process is a complex issue and there are certain factors such as social rules and cultural factors which also play a role in the process. MW6 mentioned, "it is very complex, when does the women want to come, and what does the women prefer as her midwife: is it a woman from her tribe or another? Furthermore, midwives in certain regions are treated as low level worker and in the end lower people might choose to go there and rich people from upper caste might want to prefer to go to a doctor".

\section{DESIGN IMPLICATIONS AND IMPLEMENTATION}

\section{A. Design implications and distilled design requirements}

Here we present the key design requirements that are distilled from the interviews. A diagnostic support system should be easy to use by community health workers with minimal training. It should have short answering time and can classify different diseases for pregnant women with high accuracy. The key actions to be supported are a) recording and reporting of vital information such as store and display patient information, store disease history and routine measurement of blood pressure, weight, glucose and other information by using point of care testing devices, b) diagnostics such as analysis and generating diagnosis report, classify different diseases and analyze risk factors, c) consultation such as provide referral information and provide advice on vitamin supplements, required exercise and avoiding risky behaviors, etc. Furthermore, guidelines for designing for low literate users were also consulted in addition to our design requirements [13].

\section{B. Workflow of the proposed system}

We have adopted the healthcare model practiced in the Netherlands where pregnant women come to a midwifery center for antenatal visits [9]. The community health workers in a low resource region will help to do patient registration and measure vital information along with self-reported problems such as bleeding, fever, etc. and disease history such as high blood pressure and diabetes, etc. The gathered information will be added to the proposed diagnostic system. Furthermore, the proposed diagnostic system would record each visit and in total 4 visits will be advised all over nine months. World Health Organization (WHO) also recommends at least 4 antenatal visits for the safe maternal health [2]. The first visit will be in 10-12 weeks. Next visit would in $20^{\text {th }}$ weeks for a routine checkup. The third visit is on the $30^{\text {th }}$ and final visit will be on $36-39^{\text {th }}$ week. The diagnostic system will be storing relevant diagnosis for the above mentioned weeks. If the outcome of the diagnosis from the system is critical the pregnant women will be referred to a specialist for a visit and prescribed medication and diet will be provided as necessary. If the outcome of the diagnosis is not critical, the pregnant woman will be provided advice based one the diagnosis such as recommended exercises and general tips to get fit etc.

\section{Implementation of the first prototype}

The diagnostic support system has a database where demographic information, visits to the primary healthcare center information and previous pregnancy details are stored. The graphical user interface part is used for accessing information. The front-end design utilizes visual programing and for the backend database design MySQL sever has been chosen due to its robustness. An automated algorithm analyzes risk factors and provide decisions. Furthermore, we have chosen to use windows machine due to its availability and lesser price in low-resource environments. Overall, our antenatal diagnostic system support the rural expectant mothers based on local contextual factors such as primary

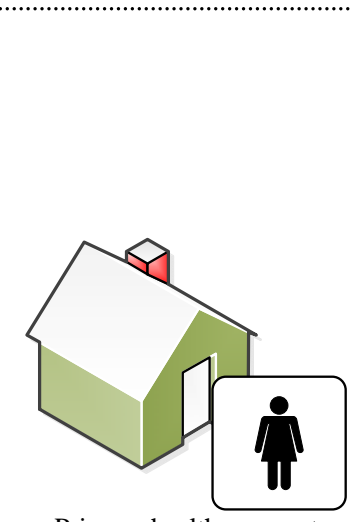

Primary healthcare center
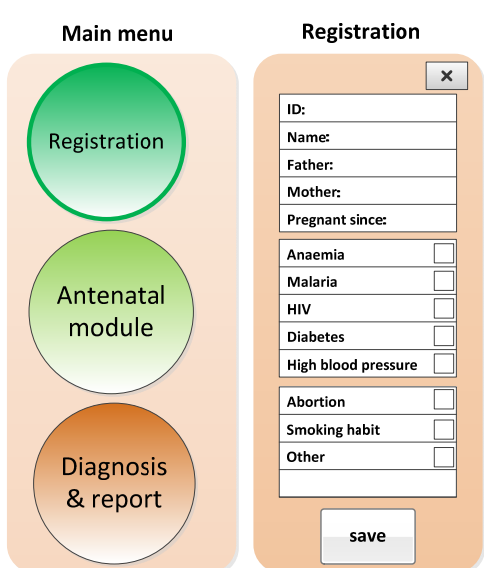

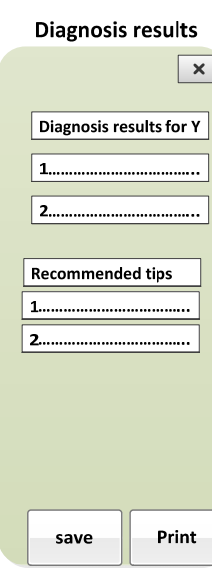

Fig. 1. The above diagram shows the basic menu structure of the proposed system. The village health workers will provide prenatal care support to pregnant women with the help of the proposed system. 
healthcare needs, most commonly occurring diseases, etc. during the pregnancy period. At present since we have adopted the model where pregnant women will visit the primary healthcare center we have decided to use a standalone computer to implement the diagnostic system. However, we also decided to implement a light version of the system suitable to use in mobile phones. This will help in case someone is ill and the rural health worker needs to visit her and they can access and update patient data on the spot. Furthermore, when required the local hospital can sync the patient data when required for an expert advice.

\section{Feedback from the midwives and gynaecologists}

The design requirements from the interviews were discussed in the design team and verified with gynecologists and midwives. Three midwives and two gynecologists were asked to evaluate the conceptual design of the diagnostic system. Each screen of the proposed diagnostic system were explained and an interaction video was shown about the concept. The feedback session was exploratory and the objective was to find out the potential of the proposed system. Some of the main point of interests were whether the system would be understandable, are the key features included, will it be a reliable system and whether it will be too complicated to operate in a low resource setting. We received positive feedback on the above mentioned aspects and some criticism which are explained below:

- Midwives confirmed that the key basic features were incorporated in the system. However, there should have provision to upgrade the system functionalities as necessary.

- The system should have additional support for the rural health workers so that they can get extra information when required. Therefore, a local corpus of relevant knowledge regarding the diagnosis would be helpful in addition to typical diagnostic results.

- The system should be implemented in a local language and all the diagnosis results should be understandable by the rural health workers.

- It would be helpful if reminders are sent to the pregnant women to visit the healthcare center for example via text messaging for the follow up visits.

- It would be helpful for both the rural health workers and the pregnant women if an assessment is made by the diagnostic system about the growth of the fetus over 9 months and visualize for interpretation for the peace of mind of the mothers.

\section{DISCUSSION AND FUTURE WORK}

There are a couple of challenges that need to be considered for the rural diagnostic software. One of the key factors is the implementation of the diagnostic system in cooperation with the local primary care centers. Strong cooperation is required to make the antenatal care system successful with local stakeholders such as NGOs, doctors, etc. Training to the community health workers is also an important issue. We aim to provide one to one training in addition to video training materials for the health workers. Technical infrastructure such as maintenance of the computers and electricity is also an issue since in remote areas electricity is not always available. Another key issue is the visits to the primary healthcare center. As it is already known that the number of visits to the antenatal care center is relatively low in rural areas where pregnant mothers have lack of primary education [2]. Initiative to make them motivated for the first and follow-up visits is a key aspect for the success of the proposed diagnostic system. Though we received the suggestion to send SMS for a reminder, still it will be an issue to bring them for the follow up visits due to social and cultural factors. We believe that if pregnant women are get benefited they will be willing to cooperate and participate. Our proposed design is different from the approaches described in [1, 8]. For example, in our approach a) data collection and treatment will be provided in the primary healthcare center, b) the data will be transferred to the regional hospital when required. We aim to refine the concept and build the next version of the functional prototype in coming months and improve it iteratively. Later on we would like to deploy the prototype in a rural setting in Asia for a longitudinal field test.

\section{ACKNOWLEDGMENTS}

We thank the midwives for their feedback and cooperation.

\section{REFERENCES}

[1] Alam, M., Tahmina, K., and Rubayat, K., “Assessing the scope for use of mobile based solution to improve maternal and child health in Bangladesh: A case study”, ICTD2010, University of London, 2010.

[2] WHO, UN Children's Fund, UN population Fund. Maternal mortality is 200: estimates developed by WHO, UNICEF, UNFA. Geneva: World Health Organization; 2004.

[3] Bullough, C., N. Meda, et al., "REVIEW: Current strategies for the reduction of maternal mortality", BJOG: An International Journal of Obstetrics \& Gynaecology 2005, 112(9), pp.1180-1188.

[4] Herz, B. K. and Measham, A. R.,“The safe motherhood initiative: Proposals for action”, World Bank, 1987.

[5] EDPS2000. http://www.tgfworld.org/edps2000.html

[6] Rooney, C., "Antenatal care and maternal health: how effective is it? A review of the evidence," Maternal Health and safe Motherhood Programme division of Family Heath. Geneva: WHO, 1992.

[7] Nunes, I., Choren, R. et al., "Supporting prenatal care in the public healthcare system in a newly industrialized country", International Foundation for Autonomous Agents and Multiagent Systems, 2010, pp. 1723-1730.

[8] CommCare. http://www.commcarehq.org/home/

[9] Dutch midwifery association. www. knov.nl

[10] Corbin, J., and Strauss, A. “Grounded theory research: Procedures, canons, and evaluative criteria,” Qualitative sociology, 1990, vol. 13, pp. 3-21.

[11] Ramachandran, D., V. Goswami, et al., "Research and reality: Using mobile messages to promote maternal health in rural india,” Proceedings of the 4th ACM/IEEE International Conference on Information and Communication Technologies and Development, ACM, 2010.

[12] Mobile Alliance for Maternal Action (MAMA). http://healthunbound.org/mama/

[13] Medhi, I., Patnaik, S. et al., "Designing mobile interfaces for novice and low-literacy users.” ACM Transactions on Computer-Human Interaction (TOCHI) 18(1): article 2, 28 pages, 2011. 